\title{
ENERGY EFFICIENT STRATEGIES FOR DEPLOYMENT OF A TWO- LEVEL WIRELESS SENSOR NETWORK
}

\author{
Ali Iranli, Morteza Maleki, Massoud Pedram \\ University of Southern California \\ \{iranli, morteza, pedram\}@usc.edu
}

\begin{abstract}
We investigate and develop energy-efficient strategies for deployment of wireless sensor networks (WSN) for the purpose of monitoring some phenomenon of interest in a coverage region. We first describe a two-level WSN structure where the sensors in the lower level monitor their surrounding environment and the microservers in the top level provide connectivity between the sensors and a base station. We then formulate and solve the problem of assigning positions and initial energy levels to the micro-servers and concurrently partitioning the sensors into clusters assigned to individual micro-servers so as maximize the monitoring lifetime of the two-level WSN subject to a total energy budget. This problem, called MDEA, is solved for both collinear deployment and planar deployment situations. Our experimental results show that the design and deployment of such a two-level WSN increase the network lifetime by a factor of two or more compared to a flat WSN with the same total initial energy and quality of monitoring.
\end{abstract}

\section{Categories and Subject Descriptors}

C.2.1 [COMPUTER-COMMUNICATION NETWORKS]

Network Architecture and Design.

General Terms

Algorithms, Performance, Design, Theory.

Keywords

Sensor deployment, energy efficiency, wireless sensor network.

\section{INTRODUCTION}

Wireless sensor networks (WSNs) are often used to monitor a spatially distributed phenomenon of interest. Sensors in such a network are most often battery-powered and collect a variety of data, such as sound, motion, temperature, or vibrations and transfer it up toward a base-station whose task is to aggregate and analyze the received data to determine if any action is needed. Given a fixed region of coverage and initial energy levels for all sensor nodes in the network, two figures of merit may be used to assess the effectiveness of a deployed sensor network: 1) the quality of monitoring (QoM), i.e., the accuracy or fidelity of the gathered data in a coverage region, and 2) the monitoring lifetime (MoL), i.e., the duration of time that this monitoring service is provided. Both figures of merit are functions of the sensor density and placement, sensor sampling rates (and hence average power consumption), network connectivity, and communication cost. Sensor deployment is a key network design step, which greatly influences both of these metrics. In particular, the optimal placement of the sensors from MoL viewpoint is not the same as the one that maximizes the QoM point of view. It follows that, with a preset total energy allocation to all sensor nodes in the network and a fixed coverage region for a WSN, one can trade

Permission to make digital or hard copies of all or part of this work for personal or classroom use is granted without fee provided that copies are not made or distributed for profit or commercial advantage and that copies bear this notice and the full citation on the first page. To copy otherwise, or republish, to post on servers or to redistribute to lists, requires prior specific permission and/or a fee.

ISLPED'05, August 8-10, 2005, San Diego, California, USA. Copyright 2005 ACM 1-59593-137-6/05/0008...\$5.00.
QoM for longer MoL or can achieve higher QoM with shorter MoL. It is thus interesting to study the problem of maximizing the MoL subject to a constraint on QoM.

Several architectures have been proposed for designing a sensor network. A simple architecture is flat architecture with homogenous sensor nodes where data from sensors are pushed toward a base station with multi-hop communication. In this structure, the communication burden is entirely with the sensor nodes. The second architecture is hierarchical heterogeneous architecture where the network consists of two layers. The first layer is a wireless sensor network containing clusters of sensor nodes that connect to a fixed micro-server (cluster head) whereas the second layer is a sparse network of micro-server nodes with access to more energy and better computation and communication capabilities, which provide for connectivity to base station. Micro-server nodes are added as data aggregator and local manager nodes to improve network performance.

There are operational advantages to a hierarchical heterogeneous layering that cannot be achieved with a "flat", homogeneous network of sensors, with its inherent limitations on power and processing capabilities. For instance, the relays help preserve limited battery resources of sensors by eliminating the need for sensors to monitor communications from their neighbors. In data gathering networks, the micro-server layer offers the advantage of caching and forwarding compressed data to the destination. Thus for a variety of applications, it appears that a relatively small number of higher-level network elements with access to more power and better computing and communication capabilities could greatly improve the performance of the overall system in terms of throughput, reliability, longevity, and flexibility. In flat sensor networks, data can only be forwarded by the sensor nodes in a multi-hop manner toward the base station. While, in a hierarchical network, data may be forwarded in a single-hop or multi-hop manner toward the micro-servers (cluster heads) and then aggregated and forwarded in a singlehop or multi-hop manner to the base station. It is of great interest to understand what performance gains can be achieved by the hierarchical networks. For a hierarchical network of $n$ mobile nodes and $m$ base-stations, it has been shown that if $m$ grows faster than $\sqrt{ } n$, the throughput capacity increases linearly with the number of base-stations, providing an effective improvement over a pure ad hoc network [1].

In this paper, the impact of hierarchical network architectures on the MoL of sensor networks is studied. More precisely, we study the MoL of two networks with the same total energy and QoM but different architectures, i.e. one flat and one hierarchical. In the process, we solve the problem of designing a two-level sensor network with maximum service lifetime. The constraints are a) the total initial energy of the network, $E_{\text {tot }}$, and b) the number of micro-sever nodes, $m$. The outputs are location and energy allocation for all micro-server nodes as well as the assignment of each sensor node to a unique micro-server so as to maximize the lifetime of the network. The problem is called Micro-server Deployment and Energy Allocation (MDEA).

The remainder of this paper is organized as follows.Section2 is 
prior work. Section 3 describes the problem that we will address and solutions approach for 1-D case. Section 4 extends the problem for 2-D case. Section 5 is simulation results.

\section{PRIOR WORK}

There have been a few published research results on deriving an upper bound on the service lifetime of multi-hop homogenous sensor networks. The service lifetime of a sensor network has been derived in [1][3] by multi-commodity flow mathematical programming formulation. It can be shown that given the locations of all sensors and micro-servers, the problem of finding minimum total energy subject to a constraint on network lifetime is the dual of finding the maximum network lifetime subject to constraint on the initial energy budget.

The LEACH protocol [4] is a protocol for forming clusters in a self-organized homogeneous sensor network when the base station is located far from the sensors. In LEACH some nodes are elected as cluster-heads while the other nodes communicate with the basestation through the nearest cluster-head. This protocol randomly rotates the job of cluster-head based on the node's remaining energy in order to uniformly distribute the energy consumption throughout the network.

The sensor placement (or deployment) problem for minimum total energy transmission (or maximum network lifetime) subject to coverage constraint has also been addressed in the literature. In [5], authors consider the placement problem of a given numbers of homogenous sensors, where data is aggregated at intermediate sensor nodes along the path toward the sink whereas in [6] a similar problem but with the objective of maximizing sensor network lifetime has been addressed. Both [5] and [6] provide solutions for sensors placement on a line and a special case of placement in a plane. In [7], authors calculate the optimum average energy cost for transmitting data to base-station, when three types of nodes are randomly deployed in a large area. In [8], authors consider only random deployment of senor and aggregators nodes and find node densities as well as initial energy of aggregators and sensors such that total cost of allocating energy is minimized.

\section{Problem Statement}

Consider that the boundaries of a sensor deployment region, (or coverage region) $R$, and the location of the base-station, $b$, is known. Furthermore, consider that reporting rate, $\mu$, is constant for all sensor nodes. The sensors are deployed such that a minimum required QoM inside $R$ is achieved. Moreover, assume that the deployment of sensors is uniform over $\mathbb{R}$. The definition and the calculation of QoM as a function of sensor density is given in [12].

As stated previously, sensors have limited communication and computation power. In order to reduce the communication burden of sensors, two-level hierarchical heterogeneous network architecture will be employed. The first level of this hierarchical network is sensor nodes and the second level is a network of micro-server nodes, which are more powerful than sensors in terms

of communication and computation capability. In other words, micro-servers build an overlay network on top of sensor nodes.

Each micro-server forms a cluster of sensors such that each sensor node in that cluster sends its data to the micro-server directly or by multi-hop routing through the other sensors inside the cluster. The micro-server then forward received data to the base-station by multi-hop routing through the other micro-servers inside the network (cf. Figure 1.) The problem that we address here is microserver deployment, sensor-to-micro-server assignment (clustering), and energy allocation to the micro-server nodes such that, for a given number of micro-servers and a constraint on the total network energy budget, the network lifetime is maximized. First, a $1-\mathrm{D}$ version of the problem is studied where sensors are deployed along a ray with the base station located at closed end of this ray. Possible applications of collinear deployment include sensor networks for border surveillance, highway traffic monitoring, safeguarding railway tracks, oil and natural gas pipeline protection, structural monitoring and surveillance of bridges and long hallways.

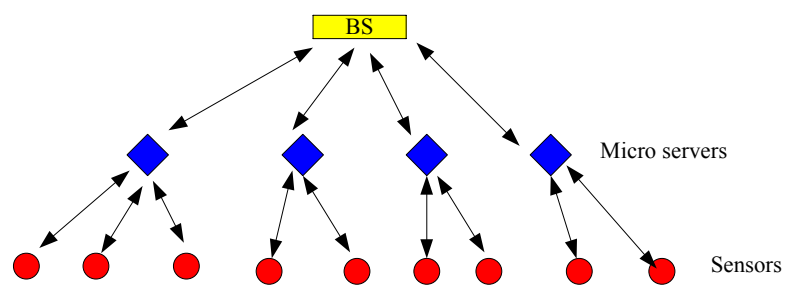

Figure 1: A two-level WSN architecture.

The key advantage of a collinear network is that it is more amenable to rigorous mathematical analysis and derivation of closed form expressions, which provides insight into understanding the more complicated case of planar sensor networks. Notice that although the analysis is initially focused on the collinear deployment of sensor nodes, we will also solve the 2-D version of this problem.

Consider a collinear network as depicted in Figure 2 where $N$ sensors with initial energy of $E_{l}$ are uniformly deployed with density $\lambda$ along the a line segment of length $L$, and therefore, the distance between two neighbor sensors is $d=1 / \lambda$. The base station is located at the origin (one end of the line segment.) We want to place $M(M<N)$ micro-servers, at positions $x_{l}, \ldots, x_{M}$ along the $x$-axis, with initial energy of $E_{2}$, such that the total network energy, i.e., $M . E_{2}+N . E_{1}$, is less than a user specified budget, $E_{t o t}$, and the lifetime of the network is maximized. Note that in collinear case the solution to the sensor clustering problem is trivial, because each sensor will consume lower energy if it connects to its closest micro-server. Also note that we allow multi-hop routing from a sensor node to its microserver and then multi-hop routing from the micro-server to the base station (but only hopping thru other micro-servers is allowed at this level, that is, once the communication is moved up to the micro-server level, it stays there.)

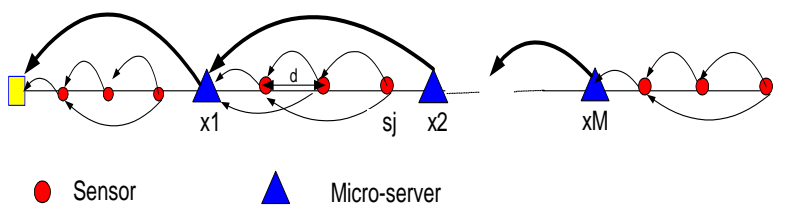

Figure 2: A collinear 2-level WSN structure.

A number of definitions for network lifetime have been proposed in the literature [9][10]. In this paper we define the network lifetime as the duration of time after which the first node (sensor or micro-server) dies out because of energy depletion. The assumption is that if any sensor node dies out after time $T_{S}$, the QoM for the application drops below the acceptable threshold. At the same time, if any micro-server dies out after time $T_{M}$, then there will be loss of connectivity between some sensors and the sink node, and hence, again QoM drops below its threshold. Now we can formally define the MDEA-1 problem.

MDEA-1 Problem: Given a line segment of length $L, N$ sensors placed at $i . L \wedge$ for $i=1, \ldots, N, M$ micro-servers, and total network energy $E_{t o t}$, the goal is to determine the initial energy levels of sensors and micro-servers, $E_{1}$ and $E_{2}$, and the locations of $M$ micro-servers, $x_{1}, \ldots, x_{M}$ so as to 


$$
\begin{aligned}
& \underset{E_{1}, E_{2}, x_{1}, \ldots, x_{M}}{\operatorname{Max}} \operatorname{Min}\left(T_{M}, T_{S}\right) \\
& \text { s.t. } N \cdot E_{1}+M \cdot E_{2} \leq E_{t o t}
\end{aligned}
$$

Implicit in this formulation is the fact that the sensor data always moves uni-directionally toward the base station. More precisely, Figure 2, data which is generated at sensor node $s_{j}$ will hop toward the left to reach the first micro server to its left and from there on it hops left in the upper level network toward the base station. We call such a routing algorithm, the "leftist routing." This should be contrasted with an alternative routing solution whereby $s_{j}$ hops toward its nearest micro-server (which in this case happens to be on its right hand side) and then hops left toward the base station. Notice that although there is tradeoff in terms of the lifetime of the sensors vs. the micro-servers depending on the choice of the routing algorithm, the leftist algorithm is always more efficient in terns of the energy dissipation per packet delivered to the base. That is why we have opted to adopt the leftist router in our analysis. To transform the MDEA-1 problem into a mathematical program, we notice that for a given deployment of micro-servers, a low-level chain of sensors, $c h_{j}$, is formed in front of each micro-server $j$. Similarly, another (high-level) chain of micro-servers is formed in front of the base station.

This simple cluster structure enables us to employ the results in [11] where the authors have shown that the optimal lifetime of a chain of $k$ sensors which are uniformly placed along a line is:

$$
T_{\text {snsr }}\left(k, E_{1}\right) \equiv \frac{E_{1}}{p_{1}}=\frac{1}{\mu \cdot e_{t}(d)} \cdot \frac{E_{1}}{(k+1) \cdot\left(1-\frac{\log (k)}{k}\right)}
$$

In this equation, $e_{t}(d)$ denotes energy per bit for data transmission over the distance between two neighboring sensors, $d$, and $E_{l}$ denotes the initial energy level of each sensor. The equation shows that the network lifetime is linearly proportional to the energy level of sensors and is inversely proportional to the size of the chain (i.e., $k$ ). Notice that, in deriving this equation, it was assumed that energy for reception and sensing are zero, therefore, this equation only gives an upper bound on the lifetime of a chain of sensors.

One can adopt a simple energy model for communication between sensors wherein energy for transmission over distance $d$ is proportional to $e_{t} \cdot d^{\beta_{1}}$ where $e_{t}$ denotes energy dissipation per meter per bit of transmitted data, $d$ denotes the distance between transmitter and receiver, and $\beta_{1}$ denotes the exponent of the path loss function for the first-level sensor network. We point out that, given a simple additive white Gaussian noise channel, the above equation is only valid under a fixed modulation scheme (e.g., the number of bits per symbol and the symbol period in a multi-QAM scheme are fixed) and a given probability of bit error. These assumptions translate to a fixed transmit bit rate for all sensors and micro-severs in the two-level network, $f^{\max }$, which is equivalent to stating that the bandwidth efficiency of all the radio transmitters is a constant. Finally, as we will see later, we will fix the total energy source in the network. So in equation (2), we ought to replace $E_{l}$ with $E_{\text {tot }} / k$. Similarly, we can write,

$$
T_{m c s r v}\left(k, E_{2}\right) \equiv \frac{E_{2}}{p_{2}(k)}=\frac{E_{2}}{f(k) \cdot\left(e_{t} \cdot l_{k}^{\beta_{2}}+e_{r}\right)}
$$

where $f(k)$ is the outgoing flow of micro-server $k$ and $l_{k}=\left|x_{k}-x_{k-1}\right|$.

Based on equations (2) and (2.a), the MDEA-1 problem can be rewritten as:

$$
\underset{E_{1}, E_{2}, l_{1}, \ldots, l_{M+1}}{\operatorname{Max}} T_{n e t} \equiv \underset{i}{\operatorname{Min}}\left(\operatorname{Min}\left(T_{\text {snsr }}\left(\lambda \cdot l_{i}, E_{1}\right), T_{m c s r v}\left(i, E_{2}\right)\right)\right)
$$

s.t.

$$
N \cdot E_{1}+M \cdot E_{2} \leq E_{t o t}
$$

$$
\begin{aligned}
& \text { s.t. } \\
& \forall i=1: M \quad f(i)=\frac{\mu \cdot \lambda \cdot l_{i+1}}{\alpha_{i}}+f(i+1) \\
& \sum_{\mathrm{i}=1: \mathrm{M}+1} l_{i}=L
\end{aligned}
$$

where the objective, $T_{n e t}$, is the network lifetime, $p(i)$ is power consumption of micro-server $i, \alpha_{i}$ denotes aggregation factor of micro-server $i$, and $f(i)$ denotes the total flow which is forwarded toward the base station by micro-server $i$. In addition, $e_{t}$ and $e_{r}$ denote the energy per meter per transmitted bit and the energy per received bit. Note that in our setup, base station is at $x_{0}=0$, micro-server $i$ is at position $x_{i}$, and the end of line segment is at position $x_{M+1}=L$.

Similar to the lower-level network, we have adopted a simple energy model for communication for the upper level network wherein energy for transmission over distance $d$ is $e_{t} \cdot d^{\beta_{2}}$ where $\left(2 \leq \beta_{2} \leq \beta_{1} \leq 5\right)$ denotes the exponent for the path loss function in the micro-server network. ${ }^{1}$ Notice also that, in our formulation, each micro-server forwards all of its data to its nearest micro-server in the direction of the base station (this is called point-to-point routing) i.e., the sensor data is relayed from one micro-server to next until it reaches the base station. This point-to-point routing also avoids the MAC layer interference, which is caused by point-to-multipoint routing. In any case, MDEA-1 can be solved by any standard mathematical program solver, including Matlab.

As an interesting variant, we consider a related problem in which, as before, the initial energy level of all of the sensors is $E_{l}$, but, this time, the initial energy levels of micro-servers are different. The rationale here is that the sensor nodes are homogenous whereas the micro-servers can have different amount of energy i.e., they have batteries with possibly different full-charge capacities and therefore micro-server $i$, has the initial energy of $E_{2}^{i}$.

MDEA-1c Problem: Given a line segment of length $L, N$ sensors placed at $i . L / \lambda$ for $i=1, \ldots, N, M$ micro-servers, and total network energy $E_{t o t}$, the goal is determine the initial energy levels of sensors and micro-servers, $E_{1}$ and $E_{2}^{i}$ 's and the locations of $M$ micro-servers, $x_{1}, \ldots, x_{M}$ so as to

$$
\begin{aligned}
& \underset{E_{1}, E_{2}^{1}, \ldots, E_{2}^{M}, l_{1}, \ldots, l_{M+1}}{\operatorname{Max}} T_{n e t}=\underset{i}{\operatorname{Min}}\left(\operatorname{Min}\left(T_{\text {snsr }}\left(\lambda \cdot l_{i}, E_{1}\right), T_{m c s r v}\left(i, E_{2}^{i}\right)\right)\right. \\
& \text { s.t. } \\
& \\
& N \cdot E_{1}+\sum_{i: 1 \ldots M} E_{2}^{i} \leq E_{\text {tot }}
\end{aligned}
$$

with the additional constraints given by (3.a).

We first state that, in the optimal solution of MDEA-1c, the lifetime of sensor nodes in any cluster (chain) is upper-bounded by the lifetime of the sensors in the longest chain connected to a micro-server. This is straight forward to check since the initial energy levels of all sensors are equal, and therefore, according to (2) the chain with maximum number of sensors, i.e., the longest chain, will have the minimum lifetime. Next we point out that, in the optimal solution of MDEA-1c, the lifetime of the longest chain is equal to the lifetime of the micro-server network. The reason is that if this is not the case, then one can decrease the

\footnotetext{
${ }^{1}$ We assume that the micro-servers use a more elaborate and thus effective channel coding technique. Therefore, their path loss exponent is lower. Of course, this comes at the cost of additional computation on the data encoder on the micro-server side and data decoder on the base station.
} 
allocated energy to micro-servers, i.e., $E_{2}^{i}$, and increase the allocated energy to the sensors, and thereby, increase the lifetime of the network. More precisely, we can write: $T_{\text {net }}^{*}=T_{\text {snsr }}^{*}\left(\lambda \cdot l_{\max }, E_{1}\right)=T_{m c s v}^{*}\left(k, E_{2}^{k}\right) \quad$ where $\forall i \quad l_{\max } \equiv l_{k} \geq l_{i}$.

Given a particular placement of the micro-servers, we can bound the lifetime of the 2-level WSN as follows:

$$
\begin{aligned}
T_{\text {net }} & \leq \frac{E_{\text {tot }}}{N \cdot p_{1}^{\max }+\sum_{j: 1 \ldots M} p_{2}(j)} \\
p_{1}^{\max } & =\mu \cdot e_{t}\left(\frac{1}{\lambda}\right) \cdot\left(\lambda \cdot l_{\max }+1\right) \cdot\left(1-\frac{\log \left(\lambda \cdot l_{\max }\right)}{\lambda \cdot l_{\max }}\right)
\end{aligned}
$$

Let $p_{2}^{\text {tot }}=\sum_{j: 1 \ldots M} p_{2}(j)$ denote the total power consumption for the $M$ micro-servers. Plugging equation (5) into objective function of MDEA-1c problem, results in the following problem statement:

$$
\begin{aligned}
& \underset{p_{2}^{\text {tot }}, l_{1}, \ldots, l_{M+1}}{\operatorname{Min}}\left(N \cdot p_{1}^{\max }+p_{2}^{\text {tot }}\right) \\
& \sum_{\mathrm{i}=1: \mathrm{M}+1} l_{i}=L \\
& l_{\max }=\operatorname{Max}_{i} l_{i} \\
& p_{1}^{\max }=\mu \cdot e_{t}\left(\frac{1}{\lambda}\right) \cdot\left(\lambda \cdot l_{\max }+1\right) \cdot\left(1-\frac{\log \left(\lambda \cdot l_{\max }\right)}{\lambda \cdot l_{\max }}\right)
\end{aligned}
$$

Note that problem (6) is in fact a Min-Max optimization since $p_{1}^{\max }$ is dependent on the maximum of $l_{i}$ 's, which are themselves variables of the minimization. To transform this Min-Max problem into a minimization problem, we note that variable $p_{1}^{\max }$ is uniquely known when we assign a value to $l_{\max }$. Furthermore, we know that that, $L /(M+1) \leq l_{\max } \leq L$, and therefore, we can sweep all values of $l_{\max }$ in the aforementioned range (in increments of $L / N)$ and, then for each value of $l_{\max }$, find the optimal solution to the following problem,

$$
\begin{aligned}
& \underset{\substack{\operatorname{Min}_{1}, \ldots, l_{M+1} \\
\text { s.t. }}}{\text { tot }} \equiv \sum_{i=1: M} p_{2}(i)=\sum_{i=1: M} f(i) \cdot\left(e_{t} \cdot l_{i}{ }^{\beta_{2}}+e_{r}\right) \\
& \forall i=1: M \quad l_{i} \leq l_{\max }
\end{aligned}
$$

with the additional constraints given by (3.a). Having found the solution to (7) for each value of $l_{\max }$, we can subsequently find the value of $l_{\max }$ which results in the optimum solution to (6).

Next we show how to solve problem statement (7). First, for simplicity, we assume $\alpha_{i}=\alpha$, for all $i$. (However, the approach can easily be generalized to handle different aggregation factors for the micro-servers.) We thus get:

$$
\begin{aligned}
& p_{2}^{\text {tot }}=e_{t} \cdot \sum_{i=1: M} f(i) \cdot l_{i}^{\beta_{2}}+\eta \cdot e_{r} \cdot \sum_{i=1: M+1} i \cdot l_{i} \\
& f(i)=\eta \cdot \sum_{j=i: M+1} l_{j} \quad \eta=\frac{\mu \cdot \lambda}{\alpha}
\end{aligned}
$$

We can write the Lagrange dual of this optimization problem:

$$
\begin{aligned}
\underset{v>0, l_{1}, \ldots, l_{M+1}}{\operatorname{Min}} p_{2}^{t o t}(v) & =e_{t} \sum_{i=1: M} f(i) \cdot l_{i}{ }^{\beta_{2}}+\eta \cdot e_{r} \cdot \sum_{i=1: M+1} i \cdot l_{i} \\
& -v \cdot\left(\left(\sum_{i=1: M+1} l_{i}-L\right)+\sum_{i=1: M+1}\left(l_{i}-l_{\max }\right)\right)
\end{aligned}
$$

where $v$ is the Lagrange multiplier. The last term captures the constraints on total length of sensor chains and the maximum length of each sensor chain. By solving the dual problem, we get

$$
\begin{gathered}
l_{i}^{*}=\left(\frac{v-i \cdot \eta \cdot e_{r}}{\beta_{2} \cdot e_{t} \cdot f(i)}\right)^{\frac{1}{\beta_{2}-1}} \quad \forall i=1: M \\
\sum_{i=1: M} l_{i}^{* \beta_{2}}=\frac{v-(M+1) \cdot \eta \cdot e_{r}}{e_{t}}
\end{gathered}
$$

where $l_{i}^{*}$ 's are the optimal sensor chain lengths. Note that equations (8.a) and (8.b) result in a set of non-linear equations in terms of $l_{i}^{*}$ 's because the amount of data flow through each micro-server, $f(i)$, is itself a function of $l_{i}^{*}$ 's as in equation (7.a). Another equation that we have is the following one:

$$
\sum_{i=1: M+1} l_{i}^{*}=L
$$

Equations (8.a), (8.b), and (8.c) yield $(\mathrm{M}+2)$ relations from which $l_{i}^{*}$ and $v$ can be calculated as follows. First, assuming $l_{M+1}^{*}=\theta$ values of $l_{M}, l_{M-1}, \ldots, l_{l}$ are calculated from equation (8.a) as a function of $\theta$ and $v$. Next, from equations (8.b) and (8.c), $\theta$ and $v$ are calculated.

\section{MDEA PRoblem in A PLANAR NETWORK}

MDEA problem in two dimensional case (i.e., the deployment region is a plane) is a difficult problem since in addition to sensor clustering, micro-server placement, and energy allocation, network routing itself adds a new degree of freedom. To simplify this problem, in this paper, we constrain ourselves to the case in which each sensor has direct transmission to the assigned micro-server and each micro-servers has direct transmission to the base-station. ${ }^{2}$ This simplification removes the routing variables from the clustering problem. (cf. Figure 3.)

We assume that locations of the sensors in the Euclidian space (denoted by $x\left(s_{i}\right)$ for sensor $s_{i}$ ) are known and the base-station is located at the origin. Let $\mathbf{S}=\left\{s_{1}, s_{2}, \ldots, s_{N}\right\}$ and $\mathbf{M}=\left\{m_{1}, m_{2}, \ldots\right.$, $\left.m_{M}\right\}$ denote the set of $N$ sensors and the set of $M$ micro-servers (cluster heads) in the two-level network, respectively. Furthermore, let $\mathbf{X}(\mathbf{S})=\left\{x\left(s_{1}\right), x\left(s_{2}\right), \ldots, x\left(s_{n}\right)\right\}$ and $\mathbf{X}(\mathbf{M})=\left\{x\left(m_{1}\right)\right.$, $\left.x\left(m_{2}\right), \ldots, x\left(m_{m}\right)\right\}$ denote the set of all sensor locations and micro-server locations, respectively. Let $m_{k}=\mathrm{M}\left(s_{i}\right)$ be the cluster head $m_{k}$ for sensor $s_{i}$, and $P_{t}\left(s_{i}, m_{k}\right)$ and $P_{t}\left(m_{k}, b\right)$ denote energy dissipation per bit of transmitted data from sensor $s_{i}$ to microserver $m_{k}$ and for micro-server $m_{k}$ to reach base station $b$. Define $A\left(m_{k}\right)=\left\{s_{i} \in \mathbf{S} \mid \mathrm{M}\left(s_{i}\right)=m_{k}\right\}$ and $C_{k}$ as the cardinality of set $A\left(m_{k}\right)$. As before, we have $P_{t}\left(s_{i}, m_{k}\right)=e_{t} \cdot\left|x\left(s_{i}\right)-x\left(m_{k}\right)\right|^{\beta_{1}}$ and $P_{t}\left(m_{k}, b\right)=e_{t} \cdot\left|x\left(m_{k}\right)-x(b)\right|^{\beta_{2}}$. Similarly, $P_{r}$ must be defined as the energy dissipation per received bit. Notice that $P_{t}\left(s_{i}, m_{k}\right) \cdot f\left(s_{i}\right)$ and $P_{r} f\left(s_{i}\right)$ denote the corresponding power dissipation levels. Assuming constant bandwidth efficiency for all the transmitters, it is easy to see that $f^{\max } \geq f\left(s_{i}\right)$ and $f^{\max } \geq \frac{\sum_{s_{i}: m_{k}=M\left(s_{i}\right)} f\left(s_{i}\right)}{\alpha\left(m_{k}\right)}$ must be satisfied.

${ }^{2}$ This kind of two-level WSN with single-hop routing within each level is very practical and highly efficient. Many applications that utilize a sensor network actually benefit from such a simple, yet cost-effective and energy-efficient, hierarchical structure. 


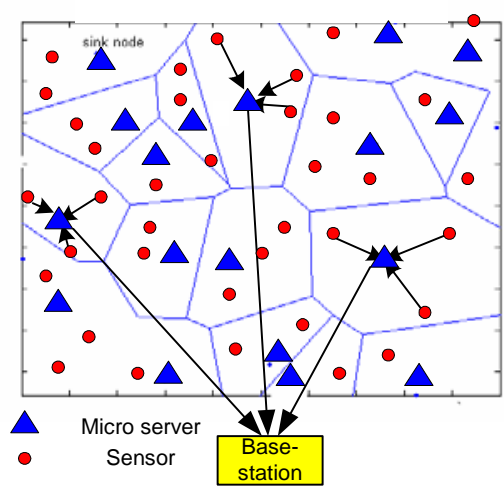

Figure 3: Single-hop routing within each level of the WSN and sensor clustering.

Let $P_{t}^{\max }$ denote the maximum transmit energy level per bit for all sensors. This means that the distance between any sensor $s_{i}$ and its cluster-head $\mathrm{M}\left(s_{i}\right)$ is upper bounded by some maximum distance, $D^{\max }$. Finally, let $b$ and $x(b)$ denote the base station and its location, and $E_{2}$ denote the energy allocated for all micro-servers $m_{k}$. With this notation, the lifetime, $T_{s_{i}}$, of sensor $s_{i}$ and the lifetime, $T_{m_{k}}$, of micro-server $m_{k}$ can be calculated as follows:

$$
\begin{gathered}
T_{s_{i}}=\frac{E_{1}}{\left(P_{t}\left(s_{i}, \mathrm{M}\left(s_{i}\right)\right)+P_{r}\right) \cdot f\left(s_{i}\right)} \\
T_{m_{k}}=\frac{E_{2}}{\left(P_{t}\left(m_{k}, b\right) \cdot \frac{1}{\alpha\left(m_{k}\right)}+P_{r}\right) \cdot \sum_{s_{i} \in A\left(m_{k}\right)} f\left(s_{i}\right)}
\end{gathered}
$$

Now, we can describe the MDEA problem more precisely.

MDEA-2 Problem: Given $\mathbf{S}, \mathbf{X}(\mathbf{S}), P_{t}^{\max }, M$, and the total network energy $E_{t o t}$, the objective is to find the sensor to microserver assignment function, $\mathrm{M}$, the locations of the micro-servers, $\mathrm{X}(\mathbf{M})$, and the sensor/micro-server energy allocations, $E_{1}$ and $E_{2}$, in such a way that:

$$
\underset{\left(\mathrm{M}(.), \mathrm{X}(.), E_{1}, E_{2}\right)}{\operatorname{Max}} \underset{m_{k} \in \mathbf{M}}{\operatorname{Min}}\left(T_{m_{k}}, \underset{S_{j} \in A\left(m_{k}\right)}{\operatorname{Min}}\left(T_{s_{j}}\right)\right)
$$

subject to:

$$
\begin{gathered}
\forall s_{i} \in \mathbf{S}, m_{k} \equiv \mathbf{M}\left(s_{i}\right) \in \mathbf{M} \\
P_{t}\left(s_{i}, m_{k}\right) \leq P_{t}^{\text {max }} \\
M \cdot E_{2}+N \cdot E_{1} \leq E_{\text {tot }}
\end{gathered}
$$

where $\alpha\left(m_{k}\right) \geq 1$ denotes the data aggregation factor for micro-server $m_{k}$. Constraint (10.a) ensures that every sensor has sufficient radio power to reach its assigned micro-server. Finally, constraint (10.b) ensures an upper bound on the total energy allocated to the network. Equations (10) can be solved by a standard mathematical programming tool.

Heuristic Solution of MDEA-2: To solve the MDEA-2 more competently, we propose a heuristic approach as follows. To begin with, we have a physical map of the sensors, $\mathrm{X}(\mathbf{S})$, in the Euclidean space, reflecting the actual layout of the sensor network on a plane. We construct a graph, $G$, whose nodes are the set of sensors, $\mathbf{S}$. The edges in $G$ are formed between pairs of sensor nodes whose Euclidean distance is less than or equal to $2 . D^{\max }$. Now, we identify the Maximum Independent Set (MIS) of this graph of size $r$. Each node in this MIS is the seed of a micro-server cluster. Clearly, $r$ should be less than or equal to $M$; otherwise, the problem has no feasible solution for the given $M$. If $r$ is less than $M$, then we will split the final clusters as will be explained in the following. In this way, we guarantee that we will always start with less than or equal to $M$ cluster seeds.

Suppose we have performed some node clustering and a partial solution, $z_{l}, \ldots, z_{M}$, has already been generated. The graph will thus comprise of these cluster nodes, $z_{k}$ 's, and a set of unassigned single nodes, $s_{i}$ 's. Next, we identify an edge connecting some $z_{k}$ to some $s_{i}$ such that $E\left(z_{k}\right)+\left(\left|\operatorname{center}\left(z_{k}, s_{i}\right)-x_{b}\right|^{\beta_{2}}-\left|x\left(z_{k}\right)-x_{b}\right|^{\beta_{2}}+P_{r}\right) \cdot f \cdot T$

has the minimum value. Here $\operatorname{center}\left(z_{k}, s_{i}\right)$ and $x\left(z_{k}\right)$ are tentative locations of the cluster heads of $z_{k} \cup\left\{s_{i}\right\}$ and $z_{k}$, respectively. Let $z_{j}$ and $s_{l}$ be such a pair. Then $s_{l}$ is collapsed into $z_{j}$ in the graph i.e., $z_{j}=z_{j} \bigcup\left\{s_{l}\right\}$. In addition, we set $x\left(z_{j}\right)=\operatorname{center}\left(z_{j}, s_{l}\right)$ and $E\left(z_{j}\right)=\left(\left|x\left(z_{j}\right)-x_{b}\right|^{\beta_{2}}+P_{r} \cdot\left|z_{j}\right|\right) \cdot f \cdot T$. This process is continued until all nodes are assigned to some cluster.

The only remaining question is how to calculate the center(.) for a set of sensor nodes $\left\{s_{u}, s_{v}, s_{w}, \ldots\right\}$.If $\beta_{2}=2$, center(.) is in fact the center of mass of identical weights placed at locations $(x, y)\left(s_{u}\right),(x, y)\left(s_{v}\right), \quad(x, y)\left(s_{w}\right), \quad \ldots \quad$ i.e., $\operatorname{center}_{x}\left(s_{u}, s_{v}, s_{w}, \ldots\right)=\frac{1}{U} \cdot \sum_{v=1 \ldots U} x\left(s_{v}\right)$, center $_{y}\left(s_{u}, s_{v}, s_{w}, \ldots\right)=\frac{1}{U} \cdot \sum_{v=1 \ldots U} y\left(s_{v}\right)$. For other values of $\beta_{2}$, a similar center of mass solution can be obtained (but solved with respect to the appropriate $\beta_{2}$-norm.) This choice reflects our desire to produce equal radio communication costs from the sensors to the cluster heads.

If the number of final clusters is smaller than the available number of micro-servers, $M$, we will first sort the clusters based on descending order of their diameter, i.e., the maximum distance of sensors in each cluster from its cluster head. Next, we assign one extra micro-server to each cluster starting from top of the list until we use all of available micro-servers. Then, we run the aforementioned greedy approach to find the new clusters.

\section{SimUlATION RESULTS}

We performed a number of simulations to assess the effectiveness of proposed solutions. In particular, we compared the lifetime of a 2-level WSN with that of a flat network. In designing the 2-level network, we employed the strategies provided above for solving MDEA-1 and MDEA-2 problems. The sensor locations were generated randomly by using a uniform distribution. They were kept unchanged throughout the simulations. We set $e_{r}=e_{t}=0.1$.

In the first simulation, we set $\lambda^{-1}=1.5$ and $\alpha=1$ (no data aggregation at the micro-servers), $\beta_{1}=2.5$ and $\beta_{2}=2$ (i.e., we assumed different channel coding efficiencies for the two levels of the WSN.) We solved MDEA-1 problem stated in equations (3) using Matlab and measured lifetimes of the flat and 2-level WSNs for a given number of micro-servers and a fixed per-node energy, $\mathrm{E}_{0}$. This means that the total energy of the flat network is $E_{t o t}=N . E_{0}$. We used the same total energy for the 2-level network of $\mathrm{N}$ sensors and $\mathrm{M}$ micro-servers, i.e., in this case $E_{\text {tot }}=N \cdot E_{1}+M \cdot E_{2}$. We report results for $\mathrm{N}=25$ and $\mathrm{N}=50$ in Figure 4 . As can be seen, having allocated the same total energy, the two-level network lifetime is higher than that of the flat network. Furthermore, for any network size, there is a particular number of micro-servers for which the lifetime of 2-level network is maximized. Finally as the network size increases, the network lifetime decreases (the total length of the line segment, 
$\mathrm{L}$, is fixed.) In this simulation, we also solved MDEA-1c

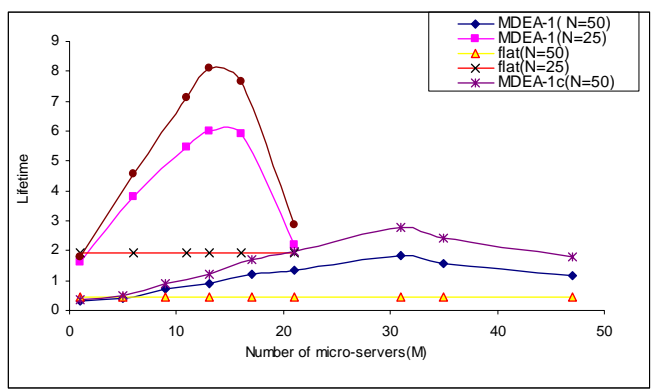

Figure 4: Collinear WSN lifetime vs. micro-server count.

problem by using the iterative Lagrange dual approach. As expected the lifetime of WSN with fixed $E_{1}$, but variable $E_{2}{ }_{2}$, , is higher than that with fixed $E_{1}$ and fixed $E_{2}$.

For the second and third simulations, we set $\beta_{1}=\beta_{2}=2$ and $\lambda^{-1}=1$. We compared the lifetime of flat network with the lifetime of 2level networks. As before, we allocated the exact same total energy $\underline{E}_{\text {tot }}$ to the flat and 2-level networks. For the flat network, we assume optimum routing so that the maximum lifetime can be achieved as indicated in equation (2). For the 2-level network, we find the optimum number of micro-servers, their locations, and their initial energy levels (for both MDEA-1 and MDEA-1c formulations), which yield the maximum lifetime. Figure 5 shows the results of the comparison. Notice that as the network size increases, the effectiveness of 2-level network in extending the network lifetime increases. In addition as can be shown in the figure, aggregation can greatly improve the effectiveness of the micro-servers. Finally, 2-level network with fixed E2 assignment for the micro-servers only outperforms flat network for large values of $\mathrm{N}$. However 2-level network with continuous assignment of energy levels to micro-servers always beats the flat network in terms of the network lifetime.

To evaluate our method for solving the MDEA-2 problem, we considered a $50 \times 50 \mathrm{~m}^{2}$ square-shaped deployment region where the base is located at center of region. We used a Poisson process to deploy sensors in the given region [12].

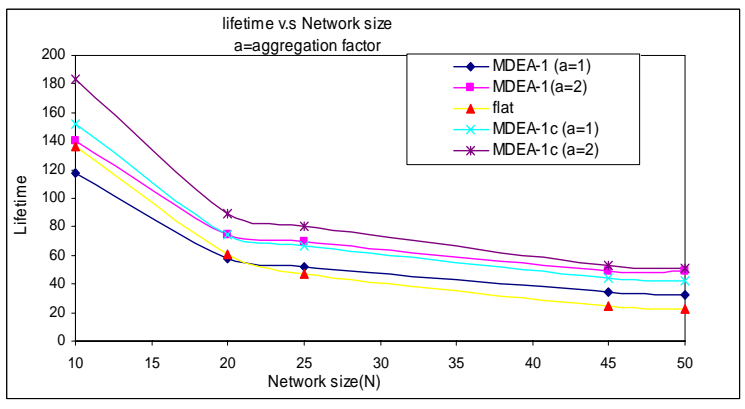

Figure 5: Lifetime comparison of 2-level and flat WSNs.

We then measured the lifetime of the 2-level network with density parameter of 0.1 and compared it with the lifetime of a flat network wherein each sensor sends its data to the base with at most two hops. In both cases, we kept $E_{\text {tot }}$ the same. Figure 6 shows the results. Locations of micro-servers are calculated in two ways: one by solving MDEA-2 problem (cf. eqn (10)) (which is called the optimal solution in the figure legend) and the other method by using the heuristic method proposed in section 4 . It can be seen that the heuristic method produces results which are close to the optimal solution with much shorter computation times (the heuristic method uses a factor of 15 lower cpu time.) Similar to the case of a collinear 2-level network, there is an optimal number of micro-servers that maximizes the lifetime of sensor network.
Finally, we point out that the 2-level network exhibits a lifetime improvement of 2.5-3X for the optimum value of micro-servers.

\section{CONCLUSION}

In this paper, we studied the operational advantages (in terms of extending the MoL of a WSN subject to a QoM constraint) of a two-level hierarchical architecture over a flat network architectures. More precisely, we formulated and solved the problem of assigning positions and initial energy levels to the micro-servers and concurrently partitioning the sensors into clusters assigned to individual micro-servers so as maximize the monitoring lifetime of the two-level WSN subject to a total energy budget. This problem, called MDEA, was solved for both collinear deployment and planar deployment situations. Experimental results showed that a factor of two or higher lifetime increase for a 2-level WSN can be achieved compared to an energy-efficient flat WSN.

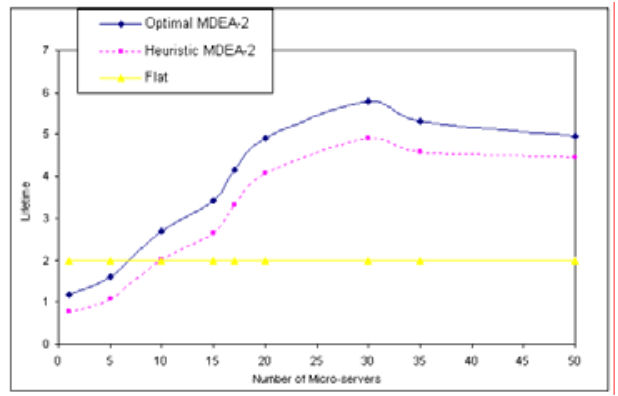

Figure 6: Lifetime comparison of 2-level and flat networks for the planar deployment of sensors in a $2500 \mathrm{~m}^{2}$ square region as a function of the micro-server count.

\section{REFERENCES}

[1] B. Liu, Z. Liu, and D. Towsley, "On the capacity of hybrid wireless networks," Proc. of Infocom, Apr. 2003.

[2] H. Chang, L. Tassiulas, "Energy Conserving Routing in Wireless Ad Hoc Networks, " Proc. of Infocom, pp. 22-31, Apr 2001.

[3] M. Bhardwaj, P. Chandrakasan, "Bounding the Lifetime of Sensor Networks Via Optimal Role Assignments," Proc. of Infocom, pp. 1587-1596, New York, June 2002.

[4] W. R. Heinzelman, A. Chandrakasan, H. Balakrishnan, "EnergyEfficient Communication Protocol for Wireless Microsensor Networks," Proc. of the 33rd Annual Hawaii International Conference on System Sciences, pp. 3005-3014, Jan. 2000.

[5] D. Ganesan, R. Cristescu and B. B-Lozano, "Power-Efficient Sensor Placement and Transmission Structure for Data Gathering under Distortion Constraints," Proc. of IPSN, Apr 2004.

[6] X. Liu and P. Mohapatra, "Energy-aware Node Placement in Wireless Sensor Networks," Proc of Globecom, 2004.

[7] V. Mhatre, C. Rosenberg, D. Kofman, R. Mazumdar and N. Shroff; "A Minimum Cost Heterogeneous Sensor Network with a Lifetime Constraint," to be appeared in proc. of IEEE Transaction on Mobile Computing, Jan. 2004.

[8] J. Seung, G. de Veciana, Su, "Minimizing energy consumption in large-scale sensor networks through distributed data compression and hierarchical aggregation," Proc. of IEEE JSAC Special Issue on Fundamental performance limits of wireless sensor networks, vol. 22, no. 6, 1130-1140, Aug. 2004.

[9] M. Maleki K.Dantu, M Pedram, "Lifetime prediction routing in mobile ad hoc networks," Proc. of WCNC, pp. 185-1190, 2003.

[10] D. Blough, Santi, "Investigating Upper Bounds on Network Lifetime Extension for Cell-Based Energy Conservation Techniques in Stationary Ad Hoc Networks," Proc. of Mobicom, Sep. 2002.

[11] A. Giridhar, P.R.Kuma, "Maximizing the Functional Lifetime of Sensor Networks," Proc. of IPSN, 2005.

[12] M.Maleki, M. Pedram, "QoM and lifetime-constrained random deployment of sensor networks for minimum energy consumption," Proc. of IPSN, 2005. 